\title{
Fiber lasers generating radially and azimuthally polarized light
}

\author{
Moti Fridman, Nir Davidson and Asher A. Friesem \\ Dept. of Physics of Complex System, Weizmann Institute of Science, Rehovo 76100, Israel
}

\author{
Galina Machavariani \\ Soreq Nuclear Research Center, Electro-Optics Division, Yavne 81800, Israel
}

\begin{abstract}
A simple, robust, and efficient method to produce either radially or azimuthally polarized output beam from a fiber laser is presented. Experimental results reveal that polarization purity of $90 \%$ or better can be obtained.
\end{abstract}

PACS numbers: 42.55.Wd, fiber lasers, 42.25.Ja Polarization

Radially and azimuthally polarized laser beams have unique properties and symmetries. For example, radially polarized light under tight focusing conditions, produces strong longitudinal electric field at the focus, while the azimuthally polarized light produces strong longitudinal magnetic field 11. Such properties are advantageous for various applications including microscopy [2, material processing [3, 4], trapping and acceleration of particles [5, 6] and laser light amplifications [7. Moreover, since the cross-section intensity distribution of radially and azimuthally polarized light have a doughnut shape, their peak intensity is significantly lower than that of a Gaussian light, so that non-linear and damage effects are reduced. Also, radially and azimuthally polarized light can propagate inside a non polarization maintaining multi-mode fiber with much less effects from birefringence than linearly polarized light $[8,9,10,11$.

Over the years, many methods have been developed in order to generate radially and azimuthally polarized light with lasers. With solid state lasers they involved phase elements 12, computer generated holograms 13 and spatially variable retarder [14. While in fiber lasers they involved an intra-cavity axicon [15] and an intracavity dual conical element [16 to produce only radially polarized light; unfortunately, these methods are highly sensitive and have low polarization purity.

In this letter, we present a highly efficient method for obtaining either radial or azimuthal polarization directly from a fiber laser. This method involves the introduction of strong losses to an unwanted polarization inside the fiber laser cavity by means of a spatially variable retarder [17] and a thin film polarizer. While this method has been successfully demonstrated with solidstate lasers 14, it was not clear whether it would be effective with fiber lasers that have relatively strong nonlinearities, high gain, and birefringence. Especially, with fiber lasers which include multimode fibers and are nonpolarization maintaining fibers.

The basic configuration for generating radially and azimuthally polarized light in a fiber laser, is schematically presented in Fig. 1. It includes ten meter long nonpolarization maintaining Erbium-doped fiber. The fiber can support few modes and has a numerical aperture of 0.07 , core diameter of $22 \mu \mathrm{m}$ and cladding diameter of

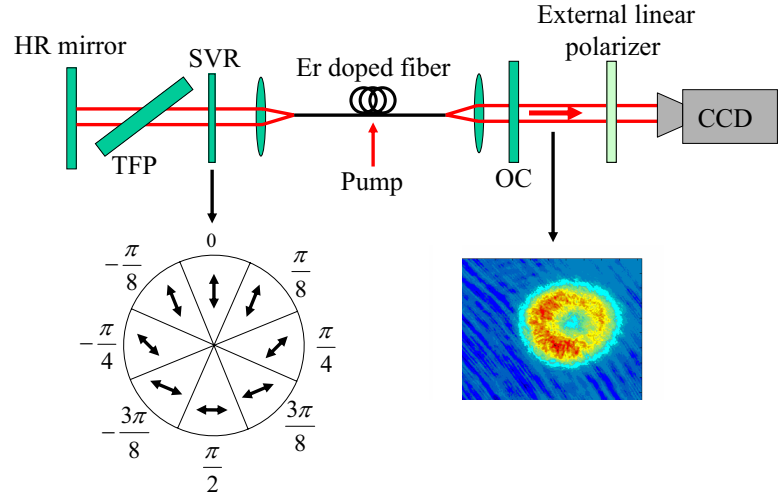

FIG. 1: Basic configuration for a fiber laser generating radially and azimuthally polarized light. TFP - thin film polarizer, OC - output coupler, HR - high reflection mirror, SVR spatially variable retarder. Lower right inset shows the typical experimental intensity distribution of the output beam with radial polarization. Lower left inset shows the orientation of the 8 sectors of the SVR.

$125 \mu \mathrm{m}$. The fiber is side pumped from both directions with two $911 \mathrm{~nm}$ multi-mode diode lasers of $100 \mathrm{~mW}$ each. The two fiber ends are cleaved at $8^{\circ}$ to suppress any reflection back into the fiber. The light emerging from one end of the fiber is collimated and propagates toward a $4 \%$ reflecting output coupler (OC), while the light emerging from the other end is collimated and propagates toward the highly reflecting (HR) back mirror. A spatially variable retarder (SVR) [17 and a thin film polarizer (TFP) are inserted between the collimating lens and the back mirror. The TFP reflects the S polarized light out of the laser so only the $\mathrm{P}$ polarized light is reflected back towards the SVR. The SVR is comprised of eight sectors each having $\lambda / 2$ retardation in different orientations, where the direction of the slow axis of each retardation plate is denoted by an arrow in Fig. 1 . When the SVR is oriented at $0^{\circ}$, it converts a linearly polarized light to radially polarized light that is reflected back into the fiber, so only radially polarized light can exist inside the fiber laser cavity. When the SVR is oriented at $45^{\circ}$ it converts the linearly polarized light to azimuthally polarized light so only azimuthally polarized light can exist in the fiber 
laser cavity. The SVR is suitable for high power and has been successfully tested up to the $\mathrm{kW}$ level 18 . Since both radially and azimuthally polarized lights have an inherent cylindrical symmetry similar to the fiber, they suffer much less from birefringence [8, 9, 10, 11].

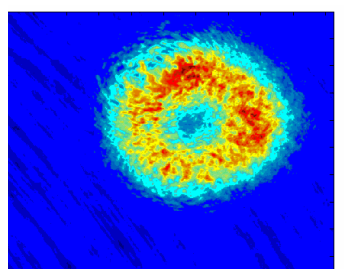

(a)

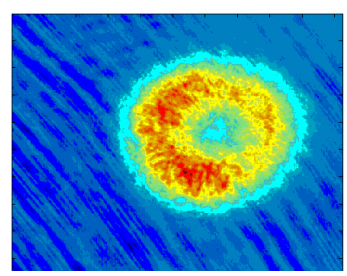

(b)
FIG. 2: Typical cross-sections of the intensity distributions of the output beam from the fiber laser. (a) Radially polarized light; (b) azimuthally polarized light.

In our experiments we first set the SVR at $0^{\circ}$ orientation so as to obtain radial polarization, and then rotated the SVR by $45^{\circ}$ to obtain azimuthal polarization. In both cases the output power of the laser was about $45 \mathrm{~mW}$. The light emerging from the fiber laser was then imaged onto a CCD camera. The detected cross-section intensity distribution of the light for both cases are presented in Fig. 2. As evident, the expected doughnut shaped distributions were obtained, and the beam quality of the outgoing beam was measured to be $M^{2}=2.05$. In order to determine the exact polarization distribution of the output light for both cases, we inserted a linear polarizer in front of the CCD camera, rotated it to several discrete orientations and detected the resulting intensity distributions.
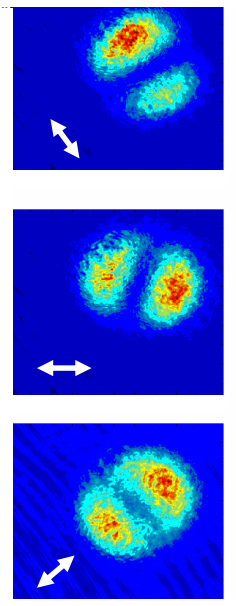
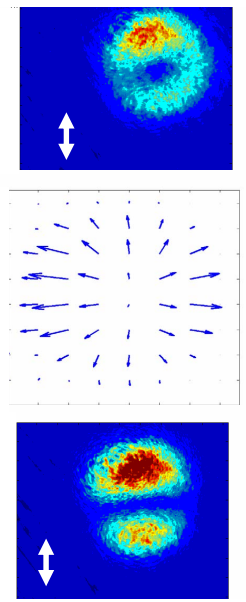
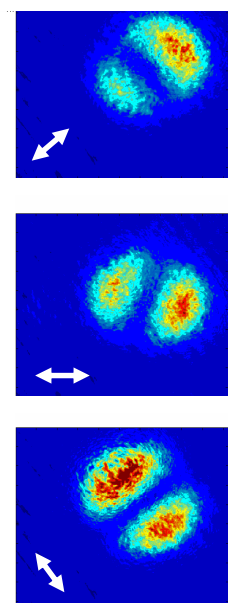

FIG. 3: Representative cross-sections of the intensity distributions of the output beam after the external linear polarizer in eight different orientations when the SVR is oriented at $0^{\circ}$. At the center, the resulting main axis of the local polarization ellipse.

The results for eight representative orientations are presented in Figs. 3 and 4. Figure 3 shows the results when the SVR is oriented at $0^{\circ}$. As evident from the detected distributions, the orientation of the common bisector of the two lobes is parallel to the direction of the external linear polarizer (denoted by the arrows), indicating radial polarization. Using these detected distributions, we calculated the local polarization at any point across the output beam [12. The results are shown in the center of Fig. 3, where the arrows indicate the direction of the main axis of the local polarization ellipse. Using these results we determined that the radial polarization purity, defined as the amount of light that would pass through a radial polarizer, is $92 \%$.
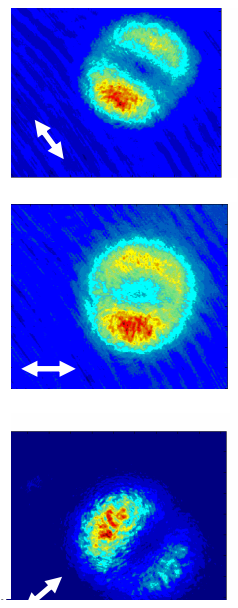
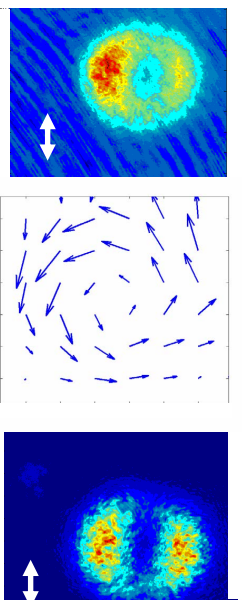
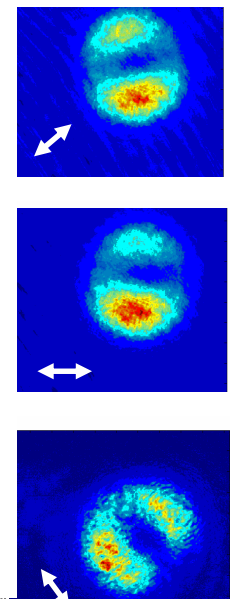

FIG. 4: Representative cross-sections of the intensity distributions of the output beam after the external linear polarizer in eight different orientations when the SVR is oriented at $45^{\circ}$. At the center, the resulting main axis of the local polarization ellipse.

Figure 4 shows the results when the SVR is oriented at $45^{\circ}$. As evident from the detected distributions, the orientation of the common bisector of the two lobes is normal to the direction of the linear polarizer (denoted by the arrows), indicating azimuthal polarization. Using these detected distributions, we calculate the local polarization at any point across the output beam. The results are shown in the center of Fig. 4, where the arrows indicate the direction of the main axis of the local polarization ellipse. We determined that the azimuthal polarization purity was $90 \%$.

In order to compare our results of radially and azimuthally polarized output light with those of linearly polarized light, we removed the SVR shown in the configuration of Fig. 1 and inserted an aperture to suppress all modes higher than the $T E M_{00}$ mode. This resulted in our laser operating with a linearly polarized Gaussian $T E M_{00}$ mode, where the polarization purity of the output light was measured to be only $70 \%$. On the other hand, when the laser operated with either radially or azimuthally polarized light, the polarization purity was $90 \%$ or better. These results indicate that redial and azimuthal polarizations are more suitable than linear polarization for multi-mode fiber lasers. 
To conclude, we presented a simple and efficient method to produce radially and azimuthally polarized light with $90 \%$ or better purity directly from fiber lasers. Although our experiments were performed with relatively low output powers, we expect that our method could be extended to higher powers which are needed in many applications.

This research was supported in part by the Binational Science Foundation.
[1] Q. Zhan and J. R. Leger. Opt. Exp., 10, 324, (2002).

[2] K. S. Youngworth and T. G. Brown. Opt. Exp., 7, 77, (2000).

[3] A. V. Nesterov and V. G. Niziev. J. Phys. D:Appl. Phys., 33, 1817, (2000).

[4] K. Venkatakrishnan and B. Tan. J. of MicroMech. and MicroEng., 16, 2603, (2006).

[5] M.O. Scully. Appl. Phys. B., 51, 238, (1990).

[6] E. J. Bochove, G. T. Moore and M. O. Scully. Phys. Rev. A., 46, 6640, (1992).

[7] I. Moshe, S. Jackel and A. Meir. Opt. Lett., 28, 807, (2003).

[8] A. Witkowska, S. G. Leon-Saval, A. Pham and T. A. Birks. Opt. Lett., 33, 306, (2008).

[9] T. Grosjean, A. Sabac and D. Courjon. Opt. Comm., 252, 12, (2005).

[10] G. Volpe and D. Petrov. Opt. Comm., 237, 89, (2004).
[11] T. Grosjean, D. Courjon and M. Spajer. Opt. Comm., 203, 1, (2002).

[12] R. Oron, S. Blit, N. Davidson, A. A. Friesem, Z. Bomzon, and E. Hasman. Appl. Phys. Lett., 77, 3322, (2000).

[13] N. Heckenberg, R. McDuff, C. Smith, and A. White. Opt. Lett., 17, 221, (1992).

[14] H. Kawauchi, Y. Kozawa, S. Sato, T. Sato, and S. Kawakami, Opt. Lett. 33, 399-401 (2008).

[15] J. -L. Li, K. -I. Ueda, M. Musha, A. Shirakawa, and Z. X. Zhang. Opt. Lett., 32, 1360, (2007).

[16] J. -L. Li, K. -I. Ueda, M. Musha, A. Shirakawa, and Z. X. Zhang. Opt. Lett., 31, 2969, (2006).

[17] G. Machavariani, Y. Lumer, I. Moshe, A. Meir, and S. Jackel Opt. Lett., 32, 1468, (2007).

[18] G. Machavariani, S. Jackel, Y. Lumer, I. Moshe, and A. Meir Proc. SPIE, 6998, 69980L (2008). 